\title{
Phylogenetic Relationship of the Longhorn Grasshopper Ruspolia differens Serville (Orthoptera: Tettigoniidae) from Northwest Tanzania Based on $18 S$ Ribosomal Nuclear Sequences
}

\author{
Nicodemus D. Matojo ${ }^{1}$ and Keneth M. Hosea ${ }^{2}$ \\ ${ }^{1}$ Department of Life Sciences, Mkwawa University College of Education (UDSM), P.O. Box 2513, Iringa, Tanzania \\ ${ }^{2}$ Department of Molecular Biology and Biotechnology, University of Dar es Salaam, Dar es Salaam, Tanzania
}

Correspondence should be addressed to Nicodemus D. Matojo; matojodn@yahoo.com

Received 16 March 2013; Revised 20 April 2013; Accepted 20 April 2013

Academic Editor: Francisco de Sousa Ramalho

Copyright (C) 2013 N. D. Matojo and K. M. Hosea. This is an open access article distributed under the Creative Commons Attribution License, which permits unrestricted use, distribution, and reproduction in any medium, provided the original work is properly cited.

\begin{abstract}
Previously, the biology of the longhorn grasshopper Ruspolia differens Serville (Orthoptera: Tettigoniidae) from northwest Tanzania was mainly inferred based on the morphological and behavioural characters with which its taxonomic status was delineated. The present study complements the previous analysis by examining the phylogenetic relationship of this insect based on the nuclear ribosomal molecular evidence. In the approach, the $18 \mathrm{~S}$ rDNA of this insect was extracted, amplified, sequenced, and aligned, and the resultant data were used to reconstruct and analyze the phylogeny of this insect based on the catalogued data.
\end{abstract}

\section{Introduction}

The longhorn grasshoppers belonging to the family Tettigoniidae, suborder Ensifera, and order Orthoptera are highly diverse insects with complex evolutionary histories $[1,2]$. The family has more than 6,400 species characterized by their long filiform antennae (which may exceed the body length), strong hind limbs, powerful chewing mouthparts, four tarsal segments, male tegminal stridulatory organs, and front tibial tympanum [3,4]. The genus Ruspolia is a group of large, elongate, cone-headed tettigoniids with yellow jaw base [58]. These insects usually occur in the grassland and open bushveld; they are active at night, mainly feed on flowers and seeds of cereals especially the millet and maize which they crack using their powerful jaws; males usually produce a very loud continuous hissing call for up to 5 minutes; nymphs hatch in 1-2 months; and they reach adult maturity in 2-3 months [9-12]. Many Ruspolia spp. have no apparent diagnostic features, and thus their taxonomy largely requires molecular evidence. However, the relationships among this genus have seldom been inferred based on molecular data $[13,14]$.
The $18 \mathrm{~S}$ rDNA is among the most widely used molecular components in phylogenetic analysis of insects $[14,15]$. Flook and Rowell [16] and Flook et al. [2] established the phylogenetic relationships among various Orthoptera groups. Liu et al. [17] described the diversity existing among members of the family Acrididae (short-horned grasshoppers). Pratt et al. [18] established the diversity among members of the families Helicidae and Rhaphidophoridae in New Zealand and Australia, whereas Robillard and Desutter-Grandcolas [19] and Danley et al. [20] inferred the phylogenetic relationship of the family Gryllidae (crickets). Molecular data of various Ruspolia spp. have been reported from different parts of the world, including Asia, Europe, and Australia [2, 18, 20]; however, there are only scanty published data from east Africa, in which there is an immense abundance of Ruspolia differens, a delicacy tettigoniid species widely known as "senene" by its Kiswahili name [6, 21].

The majority of the previous works on Ruspolia spp. have analyzed various rDNA sequences of $12 \mathrm{~S}$ and $16 \mathrm{~S}$ subunits separately without any support from $18 \mathrm{~S} \mathrm{rDNA}$ data and thus no collective results $[2,18]$. More recently, Hemp et al. [22] reported the partial sequence of $16 \mathrm{~S}$ rRNA gene of isolate 
M32 of R. differens (Accession number FM882032); however, the existing molecular records are insufficient, as they do not convey comprehensive phylogenetic relationship of this species. In this work, the phylogenetic relationship of $R$. differens from Tanzania is analysed critically based on $18 \mathrm{~S}$ rDNA nuclear sequences.

\section{Materials and Methods}

$R$. differens is a swarming insect with the tendency to shift continually within the geographical locality of its subpopulation that can cover a broad range such as Lake Victoria zone $[5,6]$. In this study, a total of 90 fresh individuals (adults) of this species were sampled randomly for molecular study. These insects were identified by their key morphological and swarming characters following the descriptions of Bailey [5]. They were collected live from different bushes in Bukoba Rural district (northwest Tanzania) during swarms of April 2009. Immediately after collection, the specimens were rinsed, sacrificed, and preserved by submerging them in $70 \%$ ethanol until analysis.

DNA extraction and PCR were conducted at the laboratory of the Department of Molecular Biology and Biotechnology (MBB) of the University of Dar es Salaam, based on the standard protocol [23]. Presence of DNA was checked on 1.5\% agarose gel electrophoresis, and viewing was done by using UV fluorescence. A pair of oligonucleotide primers used in this study was adopted from Flook et al. [2]. Each primer contained 21 nucleotide bases for each of the forward and reverse sequences. The expected sizes of PCR product were $826 \mathrm{bp}$, and the adopted primer sequences were

\section{S Fwd: GACGAAAAATAACGATACGGG} 18S Rev: CTCAATCTGTCAATCCTTCCG

Both primers were ordered in South Africa from a commercial facility, namely, INQABA Biotechnological Industries Pty Ltd. They were shipped under lyophilized state, and their sequences were defined from end $5^{\prime}$ to $3^{\prime}$ for convenient handling. Just after collection, each primer was diluted in fresh TE buffer to $200 \mathrm{pmol} / \mu \mathrm{L}$ stock solution. Prior to PCR, the stock solution was diluted to $20 \mathrm{pmol} / \mu \mathrm{L}$ working solution ready for use. All primer solutions were kept under $-4^{\circ} \mathrm{C}$ until PCR.

PCR was conducted in $25 \mu \mathrm{L}$ volume containing (i) $22 \mu \mathrm{L}$ mixture of distilled water and puReTaq "ready-to-go" beads (Lot 27-9557-01, GE Healthcare UK Limited) composed of $0.2 \mathrm{mM}$ of dNTP, $0.33 \mu \mathrm{L}$ Biotaq DNA polymerase, $1.5 \mu \mathrm{L} 10 \mathrm{x}$ $\mathrm{NH}_{4}$ reaction buffer, and $1.5 \mathrm{mM} \mathrm{MgCl}$, (ii) $1 \mu \mathrm{L}$ forward primer, (iii) $1 \mu \mathrm{L}$ reverse primer, and (iv) $1 \mu \mathrm{L}$ DNA template that was diluted in sterilized distilled water to 10 -fold.

PCR reactions were performed in a Veriti Thermal Well Cycler. Amplifications were done based on the following procedure: an initial denaturation of $2 \mathrm{~min}$ at $94^{\circ} \mathrm{C}, 30 \mathrm{~s}$ at $94^{\circ} \mathrm{C}$; annealing of $30 \mathrm{~s}$ at $49^{\circ} \mathrm{C}, 35$ cycles; extension of $1 \mathrm{~min}$ $30 \mathrm{~s}$ at $72^{\circ} \mathrm{C}$; and a final extension at $72^{\circ} \mathrm{C}$ for $3 \mathrm{~min}$ [18]. PCR products were checked on electrophoresis using $1 \%$ agarose gel with ethidium bromide staining, and the results were viewed on UV fluorescence. The PCR products and the respective primers were kept under $-20^{\circ} \mathrm{C}$ and transferred to INQABA (South Africa) where sequencing was conducted.

Prior to sequencing, the PCR products were purified using a QIAquick (QIAGEN) kit. The PCR-amplified DNA fragments were separated by electrophoresis on $1 \%$ agarose. A wide-range molecular weight DNA marker (100-bp ladder of $100-5000 \mathrm{bp}$ ) was used on each gel as the standard (INQABA Biotechnological Industries Pty Ltd). Gels were stained using ethidium bromide $(0.1 \mu \mathrm{g} / \mathrm{mL})$ for 2 hours. Sequencing was done in an ABI PRISM 3700 DNA analyser using a Big Dye Deoxy Terminator cycle-sequencing kit as per manufacturer's guidelines (Applied Biosystems Inc.).

The reverse $18 \mathrm{~S}$ rDNA sequence (partial) of $R$. differens was chosen for molecular analysis, since it was clearer. The sequence was aligned into FASTA using CLC Workbench [24], and it was "reverse complemented" using MEGA5 molecular software package (Molecular Evolutionary Genetics Analysis) ready for BLAST search (Basic Local Alignment Search Tool) [17, 25]. The BLAST search was performed online in MEGA5 against the NCBI (National Centre of Biotechnology Information) database where various complete, nearly complete, and partial sequences were widely deposited $[2,16]$. For phylogenetic analysis, the various ribosomal $18 \mathrm{~S}$ nuclear sequences matching with that of $R$. differens were first reverted to FASTA format acceptable by ClustalW alignment. The ClustalW alignment was performed in MEGA5 [26].

A section of the $R$. differens $r D N A$ sequence aligning with the dataset of significant BLAST sequences comprised $480 \mathrm{bp}$. The resultant combined dataset of the aligned sequences was selected and entered into phylogenetic analysis. Phylogenetic relationship between $R$. differens and other tettigoniids was inferred in MEGA5 based on the nuclear 18S rDNA sequence of this insect and those of its relatives that inferred 98-99\% homology in the NCBI BLAST. In this case, the Gryllidae sp. of the order Orhoptera (Accession number EU713460) was added as a chosen outgroup [2]. A phylogenetic tree was reconstructed using the Neighbor-Joining method and Maximum Parsimony method [26, 27]. All positions with less than $95 \%$ site coverage were eliminated; that is, fewer than $5 \%$ alignment gaps, missing data, and ambiguous bases were allowed at any position [26].

\section{Results}

3.1. Sampling and Molecular Methods. The sampling of $R$. differens and subsequent DNA extraction, PCR, and sequencing were successful. PCR products of approximately 850 bp were observed (Figure 1(a)).

3.2. The Nucleotide Sequence. The ascertained partial $18 \mathrm{~S}$ rDNA reverse sequence of $R$. differens was as represented in Figure 1(b) (length $841 \mathrm{bp})$.

3.3. BLAST Matches. NCBI BLAST returned a list of previously published ribosomal $18 \mathrm{~S}$ nuclear sequences significantly aligning with the $18 \mathrm{~S}$ rDNA sequence of $R$. differens (Table 1). The sequences were reported together with the respective Accession numbers, similarity scores (\%), and references. 
TABLE 1: NCBI BLAST sequences matching with $18 \mathrm{~S}$ rDNA sequence (partial) of $R$. differens from Tanzania.

\begin{tabular}{llccc}
\hline Accession number & Nearest taxon & Nucleotide sequence (partial) & Similarity score & Reference \\
\hline Z97582 & Ruspolia nitidula & 18 S rRNA & $99 \%$ & Flook and Rowell [16] \\
JF792564 & Ruspolia lineosa & 18 S rRA & $99 \%$ & Wang et al. [28] \\
JF792563 & Ruspolia dubia & $18 S$ rRNA & $99 \%$ & Wang et al. [28] \\
JF792565 & Conocephalus maculatus & $18 S$ rRNA & $99 \%$ & Wang et al. [28] \\
JF792566 & Conocephalus gladiatus & $18 S$ rRNA & $99 \%$ & Wang et al. [28] \\
JF792574 & Phyllomimus sinicus & $18 S$ rRNA & $99 \%$ & Wang et al. [28] \\
JF792573 & Orophyllus montanus & $18 S$ rRNA & $99 \%$ & Wang et al. [28] \\
EU713460 & Gryllidae sp. & $18 S$ rRNA & $98 \%$ & Flook et al. [2] \\
\hline
\end{tabular}

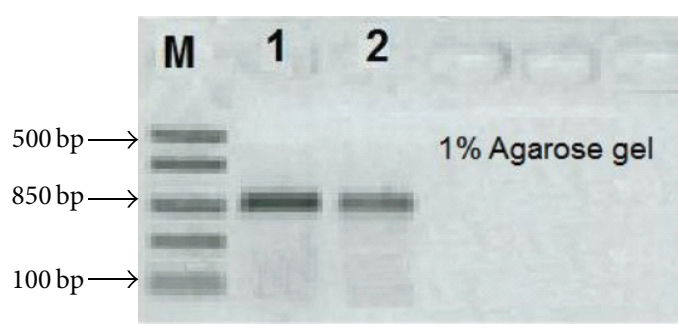

(a)

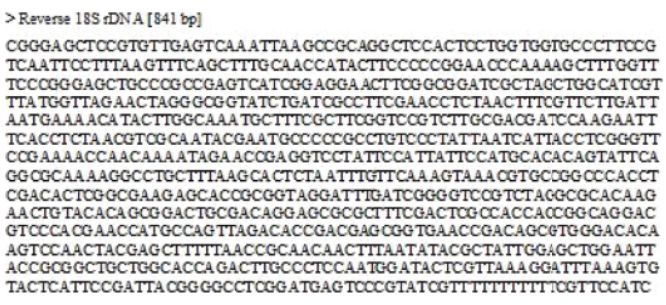

(b)

FIGURE 1: Gel photo (a) showing PCR products of partial 18S rDNA reverse sequence (b) of $R$. differens (M represents DNA size marker).

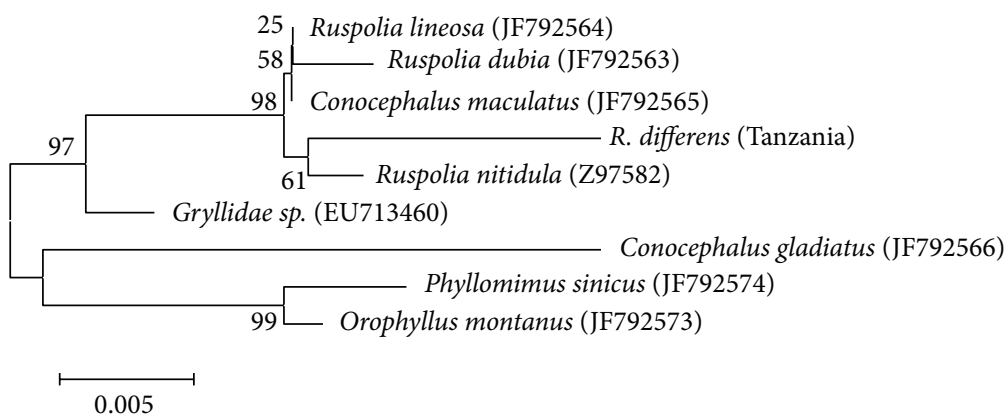

FIGURE 2: The phylogeny of $R$. differens and relatives inferred from the nuclear ribosomal 18S sequences using the Neighbor-Joining method. The tree is drawn to scale, and the branch lengths are in the same units as the evolutionary distances. Bootstraps (1050 replicates) are shown next to the branches. The optimal tree (length $=0.06974)$ is shown, with a total of 483 positions in the final dataset.

3.4. Phylogeny. Figures 2 and 3 present a phylogram of $R$. differens and relatives based on the ribosomal $18 \mathrm{~S}$ nuclear sequences inferred based on Neighbor-Joining and Maximum Parsimony methods, respectively. In the tree, $R$. differens is clustered together with all other Ruspolia spp. included in the analysis, forming a distinct clade. The closest genus shows to be Conocephalus (under 98\% bootstrap), whereas the closest relative of $R$. differens indicates to be $R$. nitidula (within 61\% bootstrap).

3.5. Relationship Patterns. Both of the resultant phylograms (Figures 2 and 3 ) have indicated that all of the comparable species are closely related to $R$. differens with the exception of Gryllidae sp. that was a chosen outgroup. However, $R$. nitidula demonstrates to be the closest species followed by $R$. lineosa and R. dubia. The closest genera to the genus Ruspolia indicates to be Conocephalus.

For comparison and verification of the relationship inferred by the nuclear ribosomal 18S dataset, the study further presents the phylogeny resulting from NCBI BLAST of 16S rRNA partial sequence of $R$. differens (Accession number FM882032) by Hemp et al. [22]. All sequences (Figure 4) inferring the homologies of $98-99 \%$ are included in the phylogeny, and there is a sequence of Gryllus campestris (Accession number Z93318) as a chosen outgroup from a distant family, namely, Gryllidae also of the order Orthoptera. Figure 4 presents the resultant phylogeny tree generated from this dataset. Likewise, $R$. differens has clustered closely with 


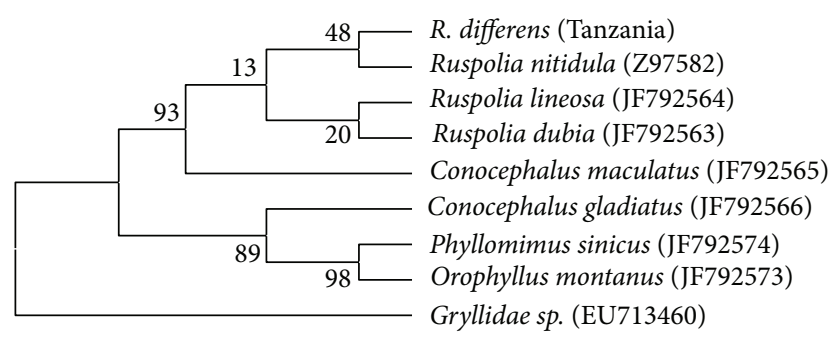

FIgURE 3: The phylogeny of $R$. differens and relatives inferred from the nuclear ribosomal $18 \mathrm{~S}$ sequences using Maximum Parsimony method. Bootstraps (1050 replicates) are shown next to the branches. The first tree of 21 most parsimonious trees (length $=47$ ) is shown, and there were a total of 445 positions in the final dataset.

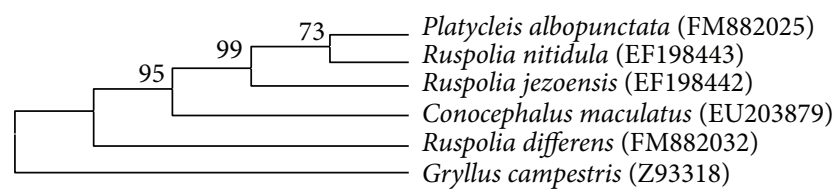

FIGURE 4: The phylogeny of $R$. differens and relatives inferred from the related catalogued nuclear ribosomal 16S sequences using Maximum Parsimony method. The most parsimonious tree (length = 424 ) is shown, and there were a total of 455 positions in the final dataset [26].

other Ruspolia spp. forming a distinct clade similar to that inferred in the previous section by the nuclear ribosomal $18 \mathrm{~S}$ molecular data. The closest genus indicates to be Conocephalus, whereas the closest species include $R$. nitidula.

\section{Discussion}

Prior to this study, the $18 \mathrm{~S}$ rDNA sequence of $R$. differens from Bukoba Tanzania was unknown, and thus it was not catalogued in any genebank. In addition, there was no detailed phylogeny reconstructed deliberately to define the relationship of this insect with other tettigoniids based on the nuclear genetic subunits. The present study has successfully used the molecular tool to isolate, sequence, and align the $18 \mathrm{~S}$ rDNA sequence of $R$. differens based on standard protocols. Then the sequence was used to analyze the phylogenetic relationship of this insect and its relatives.

The sampling of $R$. differens and subsequent molecular methods applied in this study, including DNA extraction, primer designing, PCR, sequencing, and phylogenetic analysis, were in line with those used by various other workers as they have yielded comprehensive and workable data and relationships. The intense appearance of the DNA lanes (Figure 1) indicates that the preparations were of good quality. The gel lanes have only slight smears indicating that the DNA template used was quite large and unbroken. Prospective workers can adopt similar methodology to conduct related molecular studies.

The phylogeny of $R$. differens reconstructed in this study based on $18 \mathrm{~S}$ rDNA sequence with the support from a phylogeny reconstructed using the catalogued 16S rRNA sequence [22] provides a useful picture on the phylogenetic relationship of this insect. In both cases (Figures 2-4), $R$. differens has demonstrated to be a true member of the genus Ruspolia. Its closest relative indicates to be $R$. nitidula occurring in the Palearctic region. The findings agree well with various other workers that $R$. nitidula is the closest relative and a sibling of $R$. differens $[5,6]$. Other sister species include (in brackets being their chief geographical distribution) $R$. jezoensis (Asia-temperate), R. dubia (Asia), R. lineosa (Asia), C. maculatus (Europe), and P. albopunctata (Tropical Africa, living sympatrically with $R$. differens). All these insects belong to the subfamily Copiphorinae of the family Tettigoniidae.

However, there is a clear disagreement between the present findings and those of a few other workers who diagnosed $R$. differens as $R$. nitidula $[21,29,30]$. The present findings have clearly demonstrated that these are two different species with different molecular affiliations. The shortcomings might have been contributed by the fact that, prior to this study, there were no reliable molecular data to discriminate $R$. differens from its relatives.

\section{Conclusions}

We conclude that the molecular information available in the partial $18 \mathrm{~S}$ rDNA sequence of $R$. differens concur well with those of the catalogued sequences upon which the various phylogenetic relationships of this insect and other longhorn grasshoppers (family Tettigoniidae) can be clearly inferred, and thus it is useful to incorporate these sequences in the standard molecular database for reference by subsequent workers; the relationships inferred by the $18 \mathrm{~S}$ rDNA sequence of $R$. differens are in line with those generated from the catalogued 16S rRNA sequence by Hemp et al. [22] in which it was clearly verified that the closest genera to the genus Ruspolia is Conocephalus and the closest relative to $R$. differens is $R$. nitidula; the molecular phylogeny of Ruspolia spp. and relatives is a true function of their geographical affiliations, whereas $R$. differens chiefly occurs in the African Tropics, its closest relative, that is, $R$. nitidula, is a Palearctic species occurring in North Africa, Europe, and Asia, while the rest of the other close Ruspolia spp. inferred, that is, R. dubia, $R$. lineosa, and $R$. jezoensis, are typically European and Asian species; the worldwide distribution of the Ruspolia coneheads, which is well bridged by $R$. nitidula, clearly indicates that the genus Ruspolia originated in the African Tropics before radiating to northern Africa, Europe, and Asia; the $18 \mathrm{~S}$ rDNA sequence of $R$. differens and the inferred phylogenetic relationship appear to be a useful tool for further studies on the ancestry of the tettigoniids, related taxa, and some complex phenomena such as swarming behaviour, colour polymorphism, and unique anatomy, the genes of which remain to be investigated; and $R$. differens and its sequence are of an outstanding value in research as they are capable to reveal various unresolved phylogenetic relationships. Further supportive molecular attention on $R$. differens is required, including analysis of the genes for the many unique characters of this insect. 


\section{Acknowledgments}

The authors are greatly indebted to the financial support of Mkwawa University College of Education and the University of Florida/University of Dar es Salaam Exchange Programme, as well as the technical assistance of Dr. Victor Makene and Dr. Eva Sossovelle of the Department of Molecular Biology and Biotechnology and Ms. Martha Chiduo of the Department of Zoology and Wildlife Conservation, University of Dar es Salaam.

\section{References}

[1] T. J. Walker and M. Greenfield, "Songs and systematics of Caribbean Neoconocephalus (Tettigoniidae Orthoptera)," Transactions of the American Entomological Society, vol. 109, pp. 357389, 1983.

[2] P. K. Flook, S. Klee, and C. H. F. Rowell, “Combined molecular phylogenetic analysis of the Orthoptera (Arthropoda, Insecta) and implications for their higher systematics," Systematic Biology, vol. 48, no. 2, pp. 233-253, 1999.

[3] R. F. Chapman, Insects Structure and Function, Cambridge University Press, Cambridge, UK, 1998.

[4] D. Otte, D. C. Eades, and P. Naskrecki, "Orthoptera Species File Online (2729)," 2009, http://orthoptera.speciesfile.org/.

[5] W. J. Bailey, "A review of Australian Copiphirini (Orthoptera: Tettigoniidae: Conocephalinae)," Australian Journal of Zoology, vol. 27, no. 6, pp. 1015-1049, 1979.

[6] A. W. R. McCrae, "Characteristics of swarming in the East African bush-cricket Ruspolia differens (Serville) (Orthoptera, Tettigoniidae)," Journal of the East Africa Natural History Society, vol. 178, pp. 1-8, 1982.

[7] D. C. Eades and D. Otte, "Orthoptera Species File OnlineVersion 2.0/3.5," 2009, http://orthoptera.speciesfile.org/.

[8] Matojo and J. G. Yarro, "Variability in polymorphism and sex ratio of the conehead Ruspolia differens Serville (Orthoptera: Conocephalidae) in North-west Tanzania," International Journal of Integrative Biology, vol. 9, no. 3, pp. 131-136, 2010.

[9] W. J. Bailey and D. C. F. Rentz, Eds., The Tettigoniidae: Biology, Systematics and Evolution, Springer, Berlin, Germany, 1990.

[10] B. Grzimek, D. G. Kleiman, V. Geist, and M. C. McDade, Grzimek's Animal Life Encyclopedia, Thomson-Gale, Detroit, Mich, USA, 2004.

[11] M. Picker, C. Griffiths, and A. Weaving, Field Guide to Insects of South Africa, Struik Publishers, 2004.

[12] N. D. Matojo and M. A. Njau, "Plasticity and biosystematics of swarming of the conehead Ruspolia differens serville (Orthoptera: Conocephalidae)," International Journal of Integrative Biology, vol. 9, no. 2, pp. 97-103, 2010.

[13] D. T. Gwynne and G. K. Morris, “Tettigoniidae, Katydids, Longhorned grasshoppers and bushcrickets," in Tree of Life Web Project Version, 2002.

[14] F. Legendre, T. Robillard, H. Song, M. F. Whiting, and L. Grandcolas, "One hundred years of instability in ensiferan relationships," Systematic Entomology, vol. 35, no. 3, pp. 475488, 2010.

[15] S. Kambhampati, K. M. Kjer, and B. L. Thorne, "Phylogenetic relationship among termite families based on DNA sequence of mitochondrial 16S ribosomal RNA gene," Insect Molecular Biology, vol. 5, no. 4, pp. 229-238, 1996.
[16] P. K. Flook and C. H. F. Rowell, "Inferences about orthopteroid phylogeny and molecular evolution from small subunit nuclear ribosomal DNA sequences," Insect Molecular Biology, vol. 7, no. 2, pp. 163-178, 1998.

[17] D. Liu, Z. Dong, D. Zhang et al., "Molecular phylogeny of the higher category of Acrididae (Orthoptera: Acridoidea)," Zoological Research, vol. 29, no. 6, pp. 585-591, 2008.

[18] R. C. Pratt, M. Morgan-Richards, and S. A. Trewick, "Diversification of New Zealand weta (Orthoptera: Ensifera: Anostostomatidae) and their relationships in Australasia," Philosophical Transactions of the Royal Society B, vol. 363, no. 1508, pp. 3427-3437, 2008.

[19] T. Robillard and L. Desutter-Grandcolas, "Phylogeny of the cricket subfamily Eneopterinae (Orthoptera, Grylloidea, Eneopteridae) based on four molecular loci and morphology," Molecular Phylogenetics and Evolution, vol. 40, no. 3, pp. 643-661, 2006.

[20] P. D. Danley, S. P. Mullen, F. Liu, V. Nene, J. Quackenbush, and K. L. Shaw, "A cricket gene index: a genomic resource for studying neurobiology, speciation, and molecular evolution," $B M C$ Genomics, vol. 8, article 109, 2007.

[21] J. G. Agea, D. Biryomumaisho, M. Buyinza, and N. Nabanoga, "Commercialization of Ruspolia nitidula (nsenene grasshoppers) in central Uganda," African Journal of Food Agriculture Nutrition and Development, vol. 8, no. 3, pp. 319-332, 2008.

[22] C. Hemp, K. G. Heller, S. Kehl, E. Warchaowska-liwa, W. Waegele, and A. Hemp, "The Phlesirtes complex (Orthoptera, Tettigoniidae, Conocephalinae, Conocephalini) reviewed: integrating morphological, molecular, chromosomal and bioacoustic data," Systematic Entomology, vol. 35, no. 3, pp. 554-580, 2010.

[23] F. M. Ausubel, Current Protocols in Molecular Biology, John Wiley \& Sons, New York, NY, USA, 1987.

[24] CLC bio A/S, “CLC Main Workbench-Version 6.5.1.0," Science Park Aarhus Finlandsgade 10-12 DK-8200 Aarhus, Denmark, 2011, http://www.clcbio.com/download.

[25] A. Biegert and J. Söding, "Sequence context-specific profiles for homology searching," Proceedings of the National Academy of Sciences of United States of America, vol. 106, no. 10, pp. 37703775,2009

[26] K. Tamura, D. Peterson, N. Peterson, G. Stecher, M. Nei, and S. Kumar, "MEGA5: molecular evolutionary genetics analysis using maximum likelihood, evolutionary distance, and maximum parsimony methods," Molecular Biology and Evolution, vol. 28, no. 10, pp. 2731-2739, 2011.

[27] N. Saitou and M. Nei, "The neighbor-joining method: a new method for reconstructing phylogenetic trees," Molecular Biology and Evolution, vol. 4, no. 4, pp. 406-425, 1987.

[28] X. Y. Wang, Z. J. Zhou, and Y. Huang, "The phylogenetic relationships of higher orthopteran categories inferred from $18 \mathrm{~S}$ rRNA gene sequences," Direct Submission (08-APR-2011), School of Life Sciences, Shaanxi University, Shaanxi, China, 2011.

[29] F. Dunkel, Ed., "The human use of insects as food in Uganda," Food Insects Newsletter, vol. 3, no. 8, pp. 1-12, 1995.

[30] K. Kulac, "Grasshopper Ruspolia nitidula in Uganda”, 2009, http://www.infonet-biovision.org/default/ct/123/crops. 

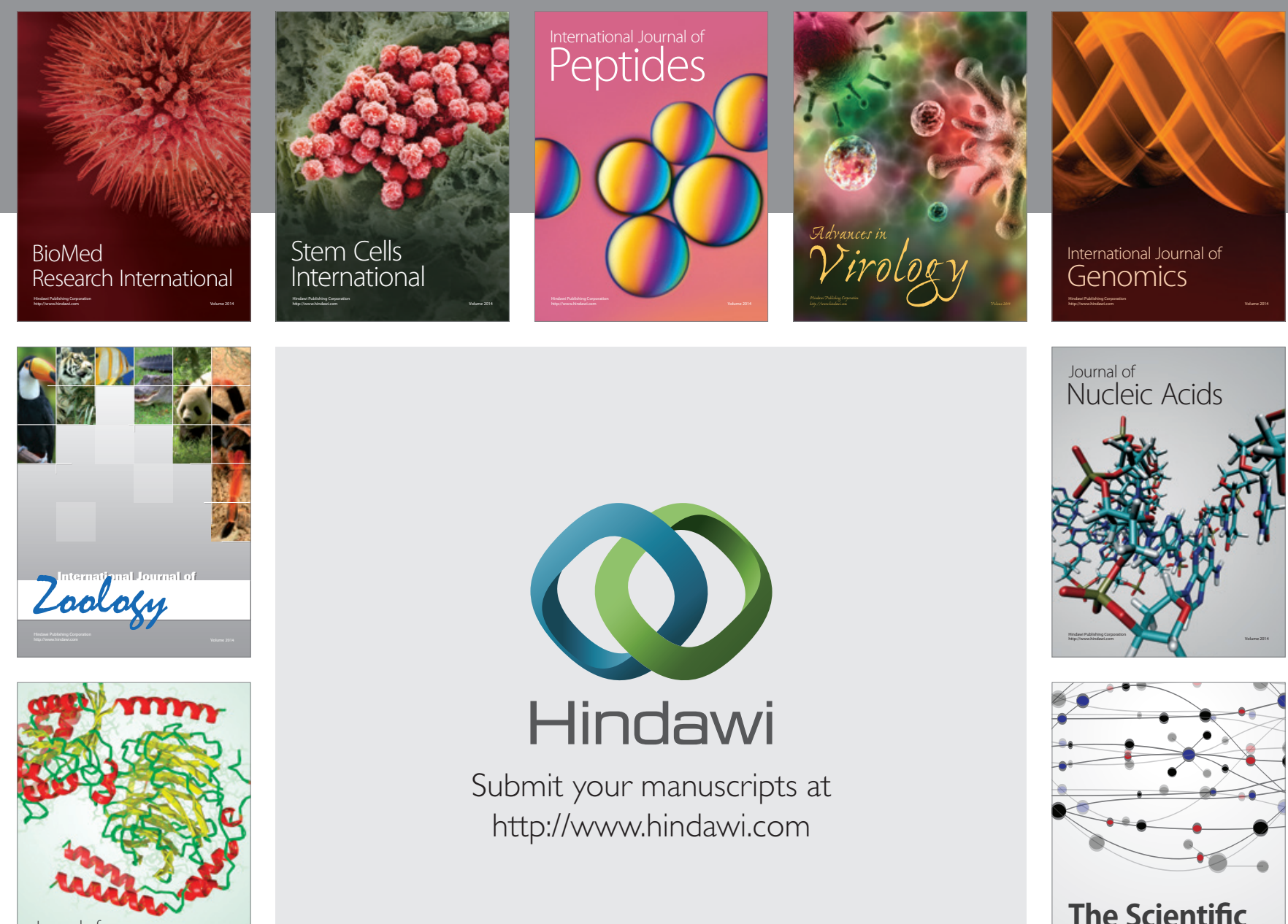

Submit your manuscripts at

http://www.hindawi.com

Journal of
Signal Transduction
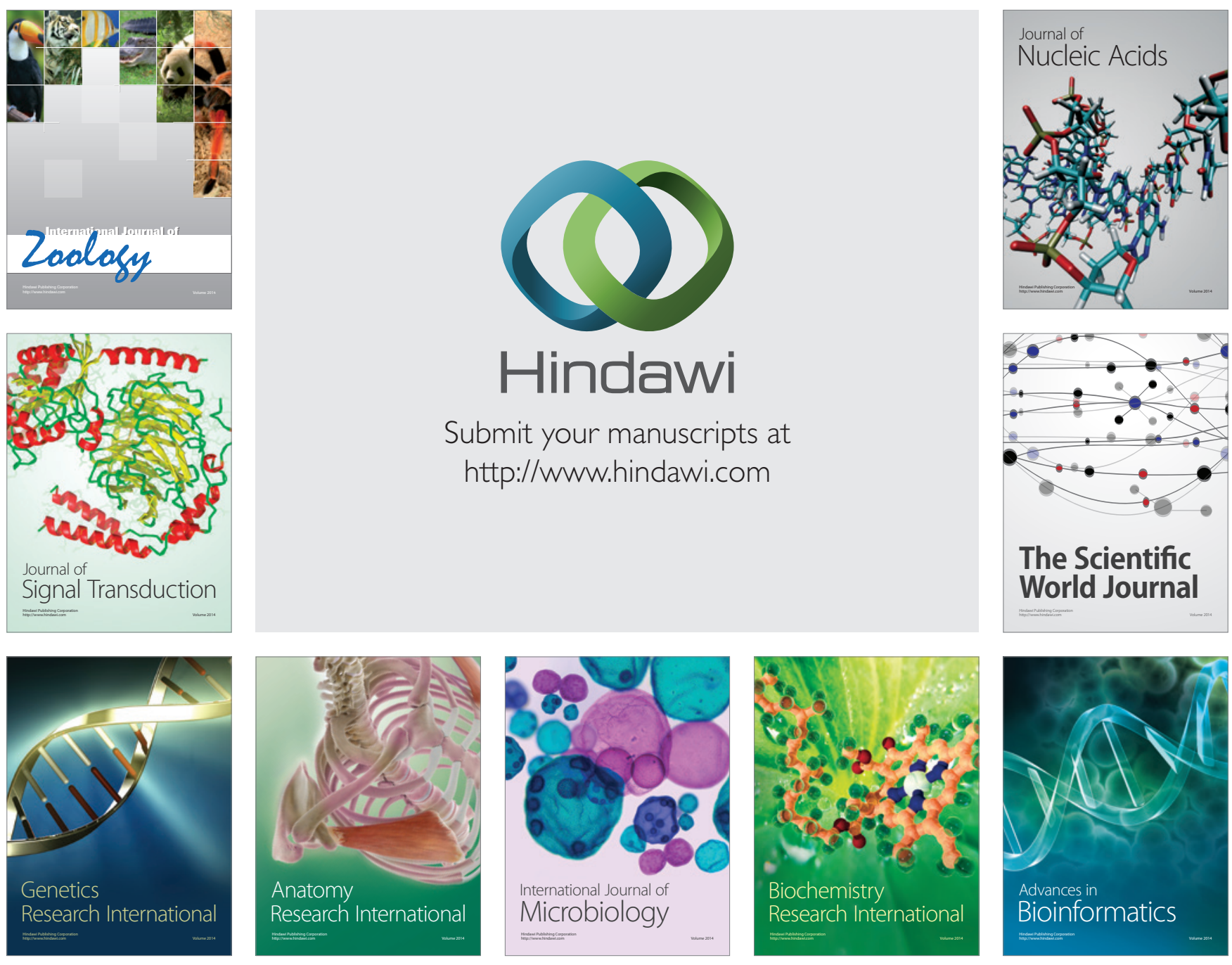

The Scientific World Journal
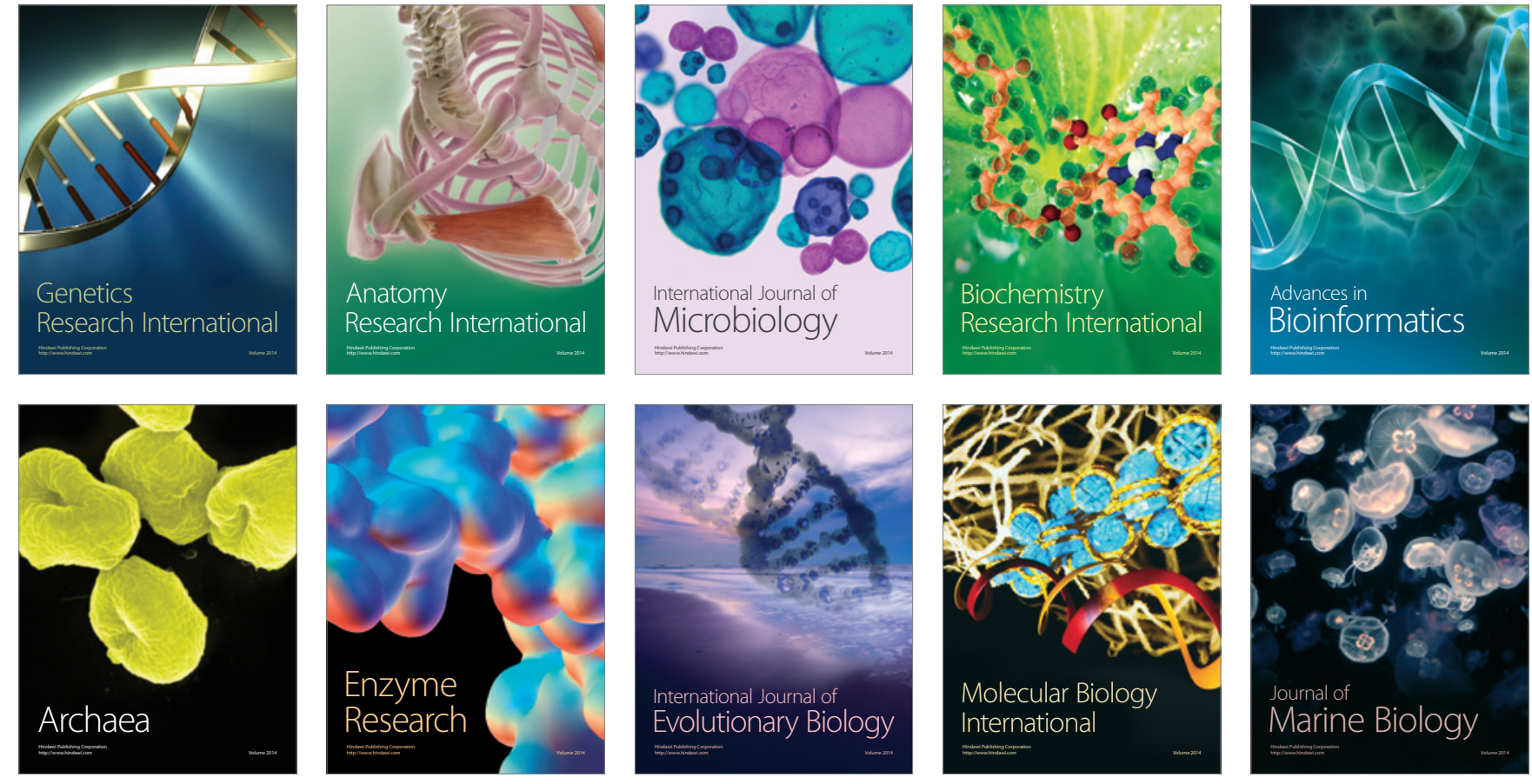